\title{
Distortion of the hyperbolicity constant of a graph
}

\author{
Walter Carballosa, Domingo Pestana, José M. Rodríguez \\ Departamento de Matemáticas \\ Universidad Carlos III de Madrid \\ Av. de la Universidad 30, 28911 Leganés, Madrid, Spain \\ wcarball@math.uc3m.es, dompes@math.uc3m.es, jomaro@math.uc3m.es
}

José M. Sigarreta

Facultad de Matemáticas

Universidad Autónoma de Guerrero

Carlos E. Adame 5, Col. La Garita, Acapulco, Guerrero, México

jsmathguerrero@gmail.com

Submitted: Oct 26, 2011; Accepted: Mar 22, 2012; Published: Mar 31, 2012

Mathematics Subject Classifications: 05C69; 05A20; 05C50

\begin{abstract}
If $\mathrm{X}$ is a geodesic metric space and $x_{1}, x_{2}, x_{3} \in X$, a geodesic triangle $T=$ $\left\{x_{1}, x_{2}, x_{3}\right\}$ is the union of the three geodesics $\left[x_{1} x_{2}\right],\left[x_{2} x_{3}\right]$ and $\left[x_{3} x_{1}\right]$ in $X$. The space $X$ is $\delta$-hyperbolic (in the Gromov sense) if any side of $T$ is contained in a $\delta$ neighborhood of the union of the other two sides, for every geodesic triangle $T$ in $X$. We denote by $\delta(X)$ the sharp hyperbolicity constant of $X$, i.e., $\delta(X):=\inf \{\delta \geq 0$ : $X$ is $\delta$-hyperbolic $\}$. The study of hyperbolic graphs is an interesting topic since the hyperbolicity of a geodesic metric space is equivalent to the hyperbolicity of a graph related to it. One of the main aims of this paper is to obtain quantitative information about the distortion of the hyperbolicity constant of the graph $G \backslash e$ obtained from the graph $G$ by deleting an arbitrary edge $e$ from it. These inequalities allow to obtain the other main result of this paper, which characterizes in a quantitative way the hyperbolicity of any graph in terms of local hyperbolicity.
\end{abstract}

Keywords: Infinite Graphs; Geodesics; Edges; Gromov Hyperbolicity.

\section{Introduction}

The study of mathematical properties of Gromov hyperbolic spaces and its applications is a topic of recent and increasing interest in graph theory; see, for instance $[1,2,3,4,6$, $7,8,10,11,15,16,17,18,19,20,21,22,24,25,26,27,29,30,31,32,33,34]$ and the references therein. 
The theory of Gromov's spaces was used initially for the study of finitely generated groups (see $[13,14]$ ), where it was demonstrated to have an enormous practical importance. This theory was applied principally to the study of automatic groups (see [23]), which play an import ant role in Sciences of Computation. Another important application of this spaces is secure transmission of information by internet (see $[15,16,17])$. In particular, the hyperbolicity also plays an important role in the spread of viruses through the network (see $[16,17]$ ). The hyperbolicity is also useful in the study of DNA data (see $[6])$.

In recent years several researchers have been interested in showing that metrics used in geometric function theory are Gromov hyperbolic. In particular, in [26, 28, 31, 33] it is proved the equivalence of the hyperbolicity of Riemann surfaces (with their Poincaré metrics) and the hyperbolicity of a simple graph; also, a classical result states that the hyperbolicity of a geodesic metric space is equivalent to the hyperbolicity of a graph related to it (see [5]), although the graph is not so simple as in the case of Riemann surfaces; hence, it is useful to know hyperbolicity criteria for graphs.

In our study on hyperbolic graphs we use the notations of [12]. We say that $\gamma$ is a geodesic if it is an isometry, i.e. $L(\gamma \mid[t, s])=d(\gamma(t), \gamma(s))=|t-s|$ for every $s, t$ in the domain of $\gamma$, where $L$ denotes length. We say that $X$ is a geodesic metric space if for every $x, y \in X$ there exists a geodesic joining $x$ and $y$; we denote by $[x y]_{X}$ or $[x y]$ any of such geodesics (since we do not require uniqueness of geodesics, this notation is ambiguous, but it is convenient). It is clear that every geodesic metric space is path-connected. If $X$ is a graph, we use the notation $[u, v]$ for the edge of a graph joining the vertices $u$ and $v$.

In order to consider a graph $G$ as a geodesic metric space, we must identify any edge $[u, v] \in E(G)$ with the real interval $[0, l]$ (if $l:=L([u, v])$ ); hence, if we consider the edge $[u, v]$ as a graph with just one edge, then it is isometric to $[0, l]$. Therefore, any point in the interior of any edge is a point of $G$. A connected graph $G$ is naturally equipped with a distance defined on its points, induced by taking shortest paths in $G$. Then, we see $G$ as a metric graph. Throughout this paper we consider graphs which are connected and locally finite (i.e., in each ball there are just a finite number of edges); we allow loops and multiple edges in the graphs; we also allow edges of arbitrary lengths. These conditions guarantee that the graph is a geodesic space (since we consider that every point in any edge of a graph $G$ is a point of $G$, whether or not it is a vertex of $G$ ).

If $X$ is a geodesic metric space and $J=\left\{J_{1}, J_{2}, \ldots, J_{n}\right\}$ is a polygon, with sides $J_{j} \subseteq X$, we say that $J$ is $\delta$-thin if for every $x \in J_{i}$ we have that $d\left(x, \cup_{j \neq i} J_{j}\right) \leq \delta$. We denote by $\delta(J)$ the sharp thin constant of $J$, i.e. $\delta(J):=\inf \{\delta \geq 0: J$ is $\delta$-thin $\}$. If $x_{1}, x_{2}, x_{3} \in X$, a geodesic triangle $T=\left\{x_{1}, x_{2}, x_{3}\right\}$ is the union of the three geodesics $\left[x_{1} x_{2}\right],\left[x_{2} x_{3}\right]$ and $\left[x_{3} x_{1}\right]$; it is usual to write also $T=\left\{\left[x_{1} x_{2}\right],\left[x_{2} x_{3}\right],\left[x_{3} x_{1}\right]\right\}$. The space $X$ is $\delta$-hyperbolic (or satisfies the Rips condition with constant $\delta$ ) if every geodesic triangle in $X$ is $\delta$-thin. We denote by $\delta(X)$ the sharp hyperbolicity constant of $X$, i.e. $\delta(X):=\sup \{\delta(T)$ : $T$ is a geodesic triangle in $X\}$. We say that $X$ is hyperbolic if $X$ is $\delta$-hyperbolic for some $\delta \geq 0$. If we have a triangle with two identical vertices, we call it a "bigon". Obviously, every bigon in a $\delta$-hyperbolic space is $\delta$-thin. It is also clear that every geodesic polygon with $n$ sides $(n \geq 3)$ in a $\delta$-hyperbolic space is $(n-2) \delta$-thin. 
The main examples of hyperbolic graphs are the trees. In fact, the hyperbolicity constant $\delta(X)$ of a geodesic metric space can be viewed as a measure of how "tree-like" the space is, since those spaces with $\delta(X)=0$ are precisely the metric trees. This is an interesting subject since, in many applications, one finds that the borderline between tractable and intractable cases may be the tree-like degree of the structure to be dealt with (see e.g. [9]).

We would like to point out that deciding whether or not a space is hyperbolic is usually extraordinarily difficult: Note that, first of all, we have to consider an arbitrary geodesic triangle $T$, and calculate the minimum distance from an arbitrary point $P$ of $T$ to the union of the other two sides of the triangle to which $P$ does not belong to. And then we have to take supremum over all the possible choices for $P$ and then over all the possible choices for $T$. Without disregarding the difficulty of solving this minimax problem, note that in general the main obstacle is that we do not know the location of geodesics in the space. Therefore, it is interesting to obtain inequalities involving the hyperbolicity constant of graphs. Since to obtain a characterization of hyperbolic graphs is a very ambitious goal, it seems reasonable to obtain criteria that guarantee the hyperbolicity.

One of the important problems in the study of any mathematical property is to determine its stability under appropriate deformations, in other words, to determine what type of perturbations preserve this property (with a quantitative control of the distortion). In the context of graphs, to delete an edge of the graph is a very natural transformation. One of the main aims of this paper is to obtain quantitative information about the distortion of the hyperbolicity constant of the graph $G \backslash e$ obtained from the graph $G$ by deleting an arbitrary edge $e$ from it. Note that this is a difficult task, since deleting an edge can change dramatically (or not) the hyperbolicity constant: on the one hand, if $C$ is a cycle graph and $e \in E(G)$, then $\delta(C)=L(C) / 4$ and $C \backslash e$ is a path graph (a tree) with $\delta(C \backslash e)=0$; on the other hand, if $G$ is any graph with a vertex $v$ of degree one and $e \in E(G)$ is the edge starting in $v$, then $\delta(G \backslash e)=\delta(G)$. However, Theorems 3.9 and 3.15 give precise upper bounds, respectively, for $\delta(G \backslash e)$ in terms of $\delta(G)$, and for $\delta(G)$ in terms of $\delta(G \backslash e)$.

These bounds allow to obtain the other main result of this paper, Theorem 4.3, which characterizes in a quantitative way the hyperbolicity of any graph in terms of local hyperbolicity. That was the idea that lead us to think of a graph $G$ as the union of some subgraphs $\left\{G_{n}\right\}_{n \geq 1}$. In order to obtain that, we call S-graph (see Section 4) to the graph $G$ obtained by "pasting" the subgraphs $\left\{G_{n}\right\}_{n \geq 1}$ "following the combinatorial design given by a graph $G_{0}$ "; Theorem 4.3 states that $G$ is $\delta$-hyperbolic if and only if $G_{n}$ is $\delta^{\prime}$-hyperbolic for every $n \geq 0$, in a simple quantitative way. Note that any graph can be viewed as a S-graph (see Section 4).

In order to prove Theorem 4.3 we need to introduce a new definition of hyperbolicity (equivalent to the previous definition) which we think that it is interesting by itself: quadrilaterals $\delta$-fine (see Section 2).

We want to remark that in the context of hyperbolic graphs it is usually not possible to obtain precise inequalities with explicit constants like the ones appearing in Theorems $3.9,3.15$ and 4.3 . 


\section{A new definition of hyperbolicity in geodesic me- tric spaces}

There are several definitions of Gromov hyperbolicity. These different definitions are equivalent in the sense that if $X$ is $\delta$-hyperbolic with respect to the definition $A$, then it is $\delta^{\prime}$-hyperbolic with respect to the definition $B$ for some $\delta^{\prime}$ (see, e.g., $[5,12]$ ).

First of all we recall the definition of fine triangles.

Definition 2.1. Given a geodesic triangle $T=\{x, y, z\}$ in a geodesic metric space $X$, let $T_{E}$ be a Euclidean triangle with sides of the same length than $T$. Since there is no possible confusion, we will use the same notation for the corresponding points in $T$ and $T_{E}$. The maximum inscribed circle in $T_{E}$ meets the side $[x, y]$ (respectively $[y, z],[z, x]$ ) in a point $z^{\prime}$ (respectively $\left.x^{\prime}, y^{\prime}\right)$ such that $d\left(x, z^{\prime}\right)=d\left(x, y^{\prime}\right), d\left(y, x^{\prime}\right)=d\left(y, z^{\prime}\right)$ and $d\left(z, x^{\prime}\right)=d\left(z, y^{\prime}\right)$. We call the points $x^{\prime}, y^{\prime}, z^{\prime}$, the internal points of $\{x, y, z\}$. There is a unique isometry $f$ of the triangle $\{x, y, z\}$ onto a tripod (a star graph with one vertex $w$ of degree 3 , and three vertices $x^{\prime \prime}, y^{\prime \prime}, z^{\prime \prime}$ of degree one, such that $d\left(x^{\prime \prime}, w\right)=d\left(x, z^{\prime}\right)=d\left(x, y^{\prime}\right)$, $d\left(y^{\prime \prime}, w\right)=d\left(y, x^{\prime}\right)=d\left(y, z^{\prime}\right)$ and $\left.d\left(z^{\prime \prime}, w\right)=d\left(z, x^{\prime}\right)=d\left(z, y^{\prime}\right)\right)$. The triangle $\{x, y, z\}$ is $\delta$-fine if $f(p)=f(q)$ implies that $d(p, q) \leq \delta$. The space $X$ is $\delta$-fine if every geodesic triangle in $X$ is $\delta$-fine.

A basic result is that hyperbolicity is equivalent to be fine:

Theorem 2.2. [12, Proposition 2.21, p.41] Let us consider a geodesic metric space $X$.

(1) If $X$ is $\delta$-hyperbolic, then it is $4 \delta$-fine.

(2) If $X$ is $\delta$-fine, then it is $\delta$-hyperbolic.

Definition 2.3. A quatripod is a double star graph, i.e, a tree with two vertices $v_{1}, v_{2}$ of degree 3 which are connected by an edge and four vertices of degree 1 two of them connected to $v_{1}$ and the other two connected to $v_{2}$. We also allow degenerated quatripods, i.e., star graphs $K_{1,4}$ (complete bipartite graph).

Remark 2.4. We also allow more degenerated quatripods, as star graphs $K_{1,3}$ (respectively, $\left.K_{1,2}\right)$. These situations correspond with quadrilaterals with several vertices repeated.

We introduce now a new definition which will play an important role in the proof of Theorem 4.3.

Definition 2.5. A geodesic metric space $X$ es $\tau$-fine for quadrilaterals if given any geodesic quadrilateral $Q=\{x, y, z, w\}$ in $X$ there exists a quatripod $\mathcal{Q}$ with vertices of degree one, $x_{0}, y_{0}, z_{0}, w_{0}$, and a map $F: Q \longrightarrow \mathcal{Q}$ such that:

i) $F(x)=x_{0}, F(y)=y_{0}, F(z)=z_{0}$, and $F(w)=w_{0}$.

ii) $F$ is an isometry between $[x y]$ and $\left[x_{0} y_{0}\right],[y z]$ and $\left[y_{0} z_{0}\right],[z w]$ and $\left[z_{0} w_{0}\right]$, and between $[w x]$ and $\left[w_{0} x_{0}\right]$.

iii) If $F(p)=F(q)$ then $d(p, q) \leq \tau$. 
This new concept of fine quadrilaterals is an equivalent definition of hyperbolicity, as Theorems 2.2 and 2.6 show.

Theorem 2.6. Let us consider a geodesic metric space $X$.

- If $X$ is $\delta$-fine for quadrilaterals, then it is $\delta$-fine (for triangles).

- If $X$ is $\delta$-fine (for triangles), then it is $2 \delta$-fine for quadrilaterals.

Proof. The first statement is direct, since a triangle is a degenerated quadrilateral with two vertices repeated. We prove now the second statement.

Given a geodesic quadrilateral $Q=\{x, y, z, w\}$, we are going to find an Euclidean quadrilateral $Q_{E}$ with sides of the same length than the sides of $Q$. Let us choose, for example, a geodesic $[x z]$ joining the vertex $x$ with the vertex $z$. We have divided in this way the quadrilateral $Q$ into two geodesic triangles $T_{1}=\{x, y, z\}$ and $T_{2}=\{x, z, w\}$. Let us consider two Euclidean triangles $T_{1, E}, T_{2, E}$ with sides of the same length than the sides of $T_{1}$ and $T_{2}$; without loss of generality we can assume that the sides of $T_{1, E}$ and $T_{2, E}$ corresponding to $[x z]$ are the real interval $[0, d(x, z)]$ in the complex plane, $T_{1, E}$ is contained in the upper halfplane and $T_{2, E}$ is contained in the lower halfplane. Since there is no possible confusion, we will use the same notation for the corresponding points in $T_{j}$ and $T_{j, E}, j=1,2$. Then $Q_{E}$ is the Euclidean quadrilateral $Q_{E}=\{x, y, z, w\}$.

Now, the maximum inscribed circle in $T_{1, E}$ meets the side $[x y]$ (respectively $[y z],[z x]$ ) in the internal point $z^{\prime}$ (respectively $\left.x^{\prime}, y^{\prime}\right)$ such that $d\left(x, z^{\prime}\right)=d\left(x, y^{\prime}\right), d\left(y, x^{\prime}\right)=d\left(y, z^{\prime}\right)$ and $d\left(z, x^{\prime}\right)=d\left(z, y^{\prime}\right)$. Similarly, the maximum inscribed circle in $T_{2, E}$ meets the side $[x z]$ (respectively $[z w],[w x]$ ) in the internal point $w^{\prime \prime}$ (respectively $x^{\prime \prime}, z^{\prime \prime}$ ) such that $d\left(x, z^{\prime \prime}\right)=d\left(x, w^{\prime \prime}\right), d\left(z, w^{\prime \prime}\right)=d\left(z, x^{\prime \prime}\right)$ and $d\left(w, x^{\prime \prime}\right)=d\left(w, z^{\prime \prime}\right)$.

There is a unique isometry $f_{1}$ of the triangle $T_{1}=\{x, y, z\}$ onto a tripod $\mathcal{T}_{1}$, with one vertex $v_{1}$ of degree 3 , and three vertices $x_{1}, y_{1}, z_{1}$ of degree 1 , such that $d\left(x_{1}, v_{1}\right)=$ $d\left(x, z^{\prime}\right)=d\left(x, y^{\prime}\right), d\left(y_{1}, v_{1}\right)=d\left(y, x^{\prime}\right)=d\left(y, z^{\prime}\right)$ and $d\left(z_{1}, v_{1}\right)=d\left(z, x^{\prime}\right)=d\left(z, y^{\prime}\right)$. As $X$ is $\delta$-fine for triangles, if $f_{1}(p)=f_{1}(q)$ then we have that $d(p, q) \leq \delta$. Similarly, there is also a unique isometry $f_{2}$ of the triangle $T_{2}=\{x, z, w\}$ onto a tripod $\mathcal{T}_{2}$ with one vertex $v_{2}$ of degree 3 , and three vertices $x_{2}, z_{2}, w_{2}$ of degree 1 , such that $d\left(x_{2}, v_{2}\right)=d\left(x, z^{\prime \prime}\right)=d\left(x, w^{\prime \prime}\right)$, $d\left(w_{2}, v_{2}\right)=d\left(w, x^{\prime \prime}\right)=d\left(w, z^{\prime \prime}\right)$ and $d\left(z_{2}, v_{2}\right)=d\left(z, w^{\prime \prime}\right)=d\left(z, x^{\prime \prime}\right)$. Again as $X$ is $\delta$-fine for triangles, if $f_{2}(p)=f_{2}(q)$ then we have that $d(p, q) \leq \delta$.

Let us consider the quatripod $\mathcal{Q}$ obtained from $\mathcal{T}_{1}$ and $\mathcal{T}_{2}$ by identifying $\left[x_{1} z_{1}\right] \subset \mathcal{T}_{1}$ with $\left[x_{2} z_{2}\right] \subset \mathcal{T}_{2}$ : i.e., $\mathcal{Q}$ is a tree with two vertices $v_{1}, v_{2}$ of degree 3 which are connected by an edge with length equal to $d\left(y^{\prime}, w^{\prime \prime}\right)$ and four vertices of degree one $x_{1}=x_{2}, y_{1}, z_{1}=$ $z_{2}, w_{2}$. Assume that $d\left(x_{1}, v_{1}\right)<d\left(x_{2}, v_{2}\right)$ (the case $d\left(x_{1}, v_{1}\right)>d\left(x_{2}, v_{2}\right)$ is similar). Then the vertices $x_{1}, y_{1}$ are connected to $v_{1}$ as in the tripod $\mathcal{T}_{1}$ and the other two $z_{2}, w_{2}$ are connected to $v_{2}$ as in the tripod $\mathcal{T}_{2}$. If $d\left(x_{1}, v_{1}\right)=d\left(x_{2}, v_{2}\right)$, then $\mathcal{Q}$ is a degenerated quatripod which is a limit case: $y^{\prime}=w^{\prime \prime}, v_{1}=v_{2}$ and $\mathcal{Q}$ is a tree with a vertex $v_{1}=v_{2}$ with degree 4.

Then there is a unique map $F$ of the quadrilateral $Q=\{x, y, z, w\}$ onto the quatripod $\mathcal{Q}$ satisfying properties $i$ ) and $i i)$ in Definition 2.5 .

Assume now that $p, q \in Q$ satisfy $F(p)=F(q)$. We have the following cases: 
i) If $F(p)=F(q)$ belongs to $\left[x_{1} z_{1}\right]=\left[x_{2} z_{2}\right]$, then, a fortiori, it must exists a point $u \in[x z]$ such that $f_{1}(p)=f_{1}(u)$ and $f_{2}(q)=f_{2}(u)$. Therefore, $d(p, u) \leq \delta$ and $d(q, u) \leq \delta$ and it follows that $d(p, q) \leq 2 \delta$ in this case.

ii) If $F(p)=F(q)$ belongs to the edge $\left[v_{1}, y_{1}\right]$, then $f_{1}(p)=f_{1}(q)$ and so $d(p, q) \leq \delta$.

iii) If $F(p)=F(q)$ belongs to the edge $\left[v_{2}, w_{2}\right]$, then $f_{2}(p)=f_{2}(q)$ and so $d(p, q) \leq \delta$.

\section{Deleting an edge}

In this section we deal with one of the main problems in the paper: to obtain quantitative relations between $\delta(G \backslash e)$ and $\delta(G)$, where $e$ is any edge of $G$. As usual, we define the graph $G \backslash e$ as the graph with $V(G \backslash e)=V(G)$ and $E(G \backslash e)=E(G) \backslash\{e\}$.

Since the proofs of these inequalities are long and technical, in order to make the arguments more transparent, we collect some results we need along the proof in technical lemmas.

Lemma 3.1. Let $G$ be any graph, $e \in E(G)$ with $G \backslash e$ connected and $x, y \in G \backslash e$. If a geodesic $\Gamma_{G}=[x y]_{G} \subset G$ contains $e$, then there exists a point $z \in \Gamma_{G \backslash e}=[x y]_{G \backslash e} \subset$ $G \backslash e$ such that the subcurve $\gamma_{x z}$ (respectively, $\gamma_{z y}$ ) contained in $\Gamma_{G \backslash e}$ and joining $x$ and $z$ (respectively, $z$ and $y$ ) is a geodesic in $G$.

Proof. Consider the points $A, B \in \Gamma_{G \backslash e}$ such that $d_{G \backslash e}(x, A)=d_{G}(x, e)$ and $d_{G \backslash e}(y, B)=$ $d_{G}(y, e)$, and choose $z$ as the midpoint of $[A, B] \subset \Gamma_{G \backslash e}$. (The points $A$ and $B$ always exist since $L\left(\Gamma_{G}\right) \leq L\left(\Gamma_{G \backslash e}\right)$.) From the fact that $\gamma_{x z} \subset \Gamma_{G \backslash e}$ and $\gamma_{z y} \subset \Gamma_{G \backslash e}$ are geodesics in $G \backslash e$, we obtain $d_{G}(z, e) \geq L([A, B]) / 2$; hence, $\gamma_{x z}$ and $\gamma_{z y}$ are geodesics in $G$.

Lemma 3.2. Let $G$ be any graph and $e \in E(G)$ with $G \backslash e$ connected. For all $x, y \in G \backslash e$, if $\Gamma_{G}=[x y]_{G}$ is a geodesic in $G$ containing e and $\Gamma_{G \backslash e}=[x y]_{G \backslash e}$ is a geodesic in $G \backslash e$, then

$$
\forall u \in \Gamma_{G \backslash e}, \exists u^{\prime} \in \Gamma_{G} \backslash e: d_{G \backslash e}\left(u, u^{\prime}\right) \leq 2 \delta(G) .
$$

Remark 3.3. In $\Gamma_{G} \backslash e$ we include the vertices connected by e.

Proof. Without loss of generality we can assume that $G$ is hyperbolic, since otherwise the inequality is direct. By Lemma 3.1 we have a point $z \in \Gamma_{G \backslash e}$ such that $T=$ $\left\{\Gamma_{G},[y z]_{G \backslash e},[z x]_{G \backslash e}\right\}$ is a geodesic triangle in $G$. Without loss of generality we can assume that $u \in[y z]_{G \backslash e}$. If $L\left([y z]_{G \backslash e}\right) \leq \delta(G)$, then there exists $u^{\prime}=y \in \Gamma_{G}$ such that $d_{G \backslash e}\left(u, u^{\prime}\right) \leq \delta(G)$. If $L\left([y z]_{G \backslash e}\right)>\delta(G)$, then we can take a point $C \in[y z]_{G \backslash e}$ such that $d_{G \backslash e}(C, z)=\delta(G)$; therefore, if $u \in[C y] \backslash\{C\}$, then the hyperbolicity of $G$ implies $d_{G}\left(u, \Gamma_{G} \cup[z x]_{G \backslash e}\right) \leq \delta(G)$; note that if $d_{G}\left(u,[z x]_{G \backslash e}\right) \leq \delta(G)$ then the geodesic $\gamma$ joining $u$ and $[z x]_{G \backslash e}$ is not contained in $G \backslash e$; in fact, $e \subset \gamma$, and since $e \subset \Gamma_{G}$ we have $d_{G}\left(u, \Gamma_{G}\right) \leq L(\gamma) \leq \delta(G)$; otherwise, $d_{G}\left(u, \Gamma_{G}\right) \leq \delta(G)$; in both cases, since 
$e \subset \Gamma_{G}$, we deduce $d_{G \backslash e}\left(u, \Gamma_{G}\right)=d_{G}\left(u, \Gamma_{G}\right) \leq \delta(G)$. Assume now that $u \in[C z]_{G \backslash e}$ (i.e., $u \in[y z]_{G \backslash e}$ with $\left.d_{G \backslash e}(u, z) \leq \delta(G)\right)$; for every $\varepsilon>0$ there exists $u_{\varepsilon} \in[y z]_{G \backslash e}$ such that $d_{G \backslash e}\left(u, u_{\varepsilon}\right) \leq \delta(G)+\varepsilon$ and $d_{G \backslash e}\left(u_{\varepsilon}, z\right)>\delta(G)$. Then there exists $u_{\varepsilon}^{\prime} \in \Gamma_{G}$ with $d_{G \backslash e}\left(u_{\varepsilon}^{\prime}, u_{\varepsilon}\right) \leq \delta(G)$ and $d_{G \backslash e}\left(u, u_{\varepsilon}^{\prime}\right) \leq 2 \delta(G)+\varepsilon$. Since $\varepsilon>0$ is arbitrary, by compactness of $\Gamma_{G}$ there exists $u^{\prime} \in \Gamma_{G}$ with $d_{G \backslash e}\left(u^{\prime}, u\right) \leq 2 \delta(G)$.

In order to finish the proof it suffices to note that if $u^{\prime}$ belongs to the interior of $e$, we can replace $u^{\prime}$ by one of the vertices joined by $e$.

We also obtain this similar result.

Lemma 3.4. Let $G$ be any graph and $e \in E(G)$ with $G \backslash e$ connected. For all $x, y \in G \backslash e$, if $\Gamma_{G}=[x y]_{G}$ is a geodesic in $G$ containing e and $\Gamma_{G \backslash e}=[x y]_{G \backslash e}$ is a geodesic in $G \backslash e$, then

$$
\forall u^{\prime} \in \Gamma_{G}, \exists u \in \Gamma_{G \backslash e}: d_{G}\left(u^{\prime}, u\right) \leq \delta(G) .
$$

Furthermore,

$$
\forall u^{\prime} \in \Gamma_{G} \backslash e, \exists u \in \Gamma_{G \backslash e}: d_{G \backslash e}\left(u^{\prime}, u\right) \leq 2 \delta(G) .
$$

Proof. Without loss of generality we can assume that $G$ is hyperbolic, since otherwise the inequalities are direct. By Lemma 3.1 we have a point $z \in \Gamma_{G \backslash e}$ such that $T=$ $\left\{\Gamma_{G},[y z]_{G \backslash e},[z x]_{G \backslash e}\right\}$ is a geodesic triangle in $G$; so (3.2) follows directly since $G$ is hyperbolic. We prove now (3.3).

Let $A$ and $B$ be the vertices of $e$, such that $[x A]_{G} \subset \Gamma_{G}$ and $[B y]_{G} \subset \Gamma_{G}$ are geodesics in $G$ with $[x A]_{G} \cap[B y]_{G}=\varnothing$. Without loss of generality we can assume that $u^{\prime} \in$ $[x A]_{G}$. If $L\left([x A]_{G}\right) \leq \delta(G)$, then there exists $u \in \Gamma_{G \backslash e}$ such that $d_{G \backslash e}\left(u^{\prime}, u\right) \leq \delta(G)$. If $L\left([A x]_{G}\right)>\delta(G)$, then let us consider the point $A^{\prime} \in[A x]_{G}$ such that $d_{G \backslash e}\left(A^{\prime}, A\right)=\delta(G)$; if $u^{\prime} \in\left[A^{\prime} x\right]_{G}$, then we have $d_{G}\left(u^{\prime}, e\right) \geq \delta(G)$ and therefore $d_{G \backslash e}\left(u^{\prime}, u\right) \leq \delta(G)$. Finally, if $u^{\prime} \in\left[A A^{\prime}\right]_{G}$, then there exits $u^{\prime \prime} \in\left[A^{\prime} x\right]_{G}$ such that $d_{G \backslash e}\left(u^{\prime}, u^{\prime \prime}\right) \leq \delta(G)$; hence, there exits $u \in \Gamma_{G \backslash e}$ such that $d_{G \backslash e}\left(u^{\prime}, u\right) \leq d_{G \backslash e}\left(u^{\prime}, u^{\prime \prime}\right)+d_{G \backslash e}\left(u^{\prime \prime}, u\right) \leq 2 \delta(G)$.

The argument in the proof of Lemma 3.4 also gives the following result.

Corollary 3.5. Let $G$ be any graph, $e \in E(G)$ with $G \backslash e$ connected, and $x, y, z \in G \backslash e$; let $T=\{[x y],[y z],[z x]\}$ be a geodesic triangle in $G$ such that $[x y]$ contains $e$ and $[y z]$, $[z x]$ do not contain e. Then

$$
\forall u^{\prime} \in[x y] \backslash e, \exists u \in[y z] \cup[z x]: d_{G \backslash e}\left(u^{\prime}, u\right) \leq 2 \delta(G) .
$$

Lemma 3.6. Let $G$ be any graph and $e \in E(G)$ with $G \backslash$ e connected. Let $T_{G}=$ $\left\{[x y]_{G},[y z]_{G},[z x]_{G}\right\}$ be a geodesic triangle in $G$ with $x, y, z \in G \backslash e$. Then $e$ is contained at most in two of the three sides of $T_{G}$.

Proof. Without loss of generality we can assume that $e=[A, B]$ is contained in $[x y]_{G}$ and $[x z]_{G}$. Since $[x y]_{G}=[x A]_{G \backslash e} \cup[A, B] \cup[B y]_{G \backslash e}$, we have $L\left([x B]_{G \backslash e}\right) \geq L\left([x A]_{G \backslash e}\right)+L(e)$ and $L\left([A y]_{G \backslash e}\right) \geq L(e)+L\left([B y]_{G \backslash e}\right)$; since $[x z]_{G}=[x A]_{G \backslash e} \cup[A, B] \cup[B z]_{G \backslash e}$, we have $L\left([A z]_{G \backslash e}\right) \geq L(e)+L\left([z B]_{G \backslash e}\right)$. Hence, $\min \{L(\gamma): \gamma$ is a path in $G$ between $y$ and $z$ with $e \subset \gamma\} \geq L(e)+d_{G \backslash e}(y, B)+d_{G \backslash e}(B, z)$; since $d_{G}(y, z) \leq d_{G \backslash e}(y, B)+d_{G \backslash e}(B, z)$, then $e$ is not contained in $[y z]_{G}$. 
Definition 3.7. We say that a subgraph $\Gamma$ of $G$ is isometric if $d_{\Gamma}(x, y)=d_{G}(x, y)$ for every $x, y \in \Gamma$.

We will need the following result (see [30, Lemma 5]).

Lemma 3.8. If $\Gamma$ is an isometric subgraph of $G$, then $\delta(\Gamma) \leq \delta(G)$.

We can prove now the following Theorem.

Theorem 3.9. Let $G$ be any graph and $e \in E(G)$ with $G \backslash e$ connected. The following inequality holds

$$
\delta(G \backslash e) \leq 5 \delta(G)
$$

Proof. Without loss of generality we can assume that $G$ is hyperbolic, since otherwise the inequality is direct. If $e=[A, B]$ and $L(e) \geq d_{G \backslash e}(A, B)$, then $G \backslash e$ is an isometric subgraph of $G$ and Lemma 3.8 gives $\delta(G \backslash e) \leq \delta(G)$. Assume now that $L(e)<d_{G \backslash e}(A, B)$.

Let us consider an arbitrary geodesic triangle $T_{G \backslash e}=\left\{[x y]_{G \backslash e},[y z]_{G \backslash e},[z x]_{G \backslash e}\right\}$ in $G \backslash e$. Let $T_{G}$ be a geodesic triangle of $G$ with the same vertices of $T_{G \backslash e}$, i.e., $T_{G}=$ $\left\{[x y]_{G},[y z]_{G},[z x]_{G}\right\}$, satisfying the following property: if $a$ and $b$ are vertices of $T_{G \backslash e}$ with $d_{G \backslash e}(a, b)=d_{G}(a, b)$, then we choose $[a b]_{G}$ as $[a b]_{G \backslash e}$. If $n$ is the number of the geodesic sides of $T_{G}$ containing $e$, then by Lemma $3.6 n$ is either 0,1 or 2 .

Case $n=0$. In this case we have $T_{G}=T_{G \backslash e}$. Let us consider any $\alpha \in T_{G \backslash e}$; without loss of generality we can assume that $\alpha \in[x y]_{G \backslash e}$.

Since $G$ is hyperbolic, there exists $\beta \in[x z]_{G \backslash e} \cup[y z]_{G \backslash e}$ such that $d_{G}(\alpha, \beta) \leq \delta(G)$. If $d_{G \backslash e}(\alpha, \beta)=d_{G}(\alpha, \beta) \leq \delta(G)$, then $d_{G \backslash e}\left(\alpha,[x z]_{G \backslash e} \cup[y z]_{G \backslash e}\right) \leq \delta(G)$. Hence, we can assume that $d_{G \backslash e}(\alpha, \beta)>d_{G}(\alpha, \beta)$; then the geodesic in $G$ joining $\alpha$ and $\beta$ contains $e$. Let $\gamma_{1}$ be the geodesic contained in $[x y]_{G \backslash e}$ joining $\alpha$ and $x$, and let $\gamma_{2}$ be the geodesic contained in $[x y]_{G \backslash e}$ joining $\alpha$ and $y$; then $\gamma_{1} \cup \gamma_{2}=[x y]_{G \backslash e}$.

If $L\left(\gamma_{1}\right) \leq 2 \delta(G)$ or $L\left(\gamma_{2}\right) \leq 2 \delta(G)$, then there exists $\beta \in\{x, y\} \subset[x z]_{G \backslash e} \cup[y z]_{G \backslash e}$ such that $d_{G \backslash e}(\alpha, \beta) \leq 2 \delta(G)$.

If $L\left(\gamma_{1}\right)>2 \delta(G)$, then consider the point $\alpha^{\prime} \in \gamma_{1}$ such that $d_{G \backslash e}\left(\alpha, \alpha^{\prime}\right)=2 \delta(G)$. Since $G$ is hyperbolic, there exists $\beta^{\prime} \in[x z]_{G \backslash e} \cup[y z]_{G \backslash e}$ such that $d_{G}\left(\alpha^{\prime}, \beta^{\prime}\right) \leq \delta(G)$. If $d_{G \backslash e}\left(\alpha^{\prime}, \beta^{\prime}\right)=d_{G}\left(\alpha^{\prime}, \beta^{\prime}\right) \leq \delta(G)$, then we conclude $d_{G \backslash e}\left(\alpha,[x z]_{G \backslash e} \cup[y z]_{G \backslash e}\right) \leq$ $3 \delta(G)$. If $d_{G \backslash e}\left(\alpha^{\prime}, \beta^{\prime}\right)>d_{G}\left(\alpha^{\prime}, \beta^{\prime}\right)$, then the geodesic in $G$ joining $\alpha^{\prime}$ and $\beta^{\prime}$ contains $e$; recall that the geodesic in $G$ joining $\alpha$ and $\beta$ contains $e$; hence, there exists a path in $G$ joining $\alpha$ and $\alpha^{\prime}$ with length less than $2 \delta(G)$, and therefore $[x y]_{G \backslash e}$ is not a geodesic in $G$. This is a contradiction, and we conclude $d_{G \backslash e}\left(\alpha,[x z]_{G \backslash e} \cup[y z]_{G \backslash e}\right) \leq$ $3 \delta(G)$.

Therefore, $\delta\left(T_{G \backslash e}\right) \leq 3 \delta(G)$ in the case $n=0$.

Case $n=1$. In this case, without loss of generality we can assume that $[x z]_{G}=[x z]_{G \backslash e}$ and $[y z]_{G}=[y z]_{G \backslash e}$. 
By Lemma 3.2, for any $\alpha_{1} \in[x y]_{G \backslash e}$ there is $\alpha^{\prime} \in[x y]_{G} \backslash e$ such that $d_{G \backslash e}\left(\alpha_{1}, \alpha^{\prime}\right) \leq$ $2 \delta(G)$. Furthermore, by Corollary 3.5 there exists $\beta_{1} \in[x z]_{G} \cup[y z]_{G}$ such that $d_{G \backslash e}\left(\alpha^{\prime}, \beta_{1}\right) \leq 2 \delta(G)$. Hence, we have $d_{G \backslash e}\left(\alpha_{1}, \beta_{1}\right) \leq 4 \delta(G)$.

Let us consider now any $\alpha_{2} \in[x z]_{G \backslash e} \cup[y z]_{G \backslash e}$; without loss of generality we can assume that $\alpha_{2} \in[y z]_{G \backslash e}$. Since $T^{\prime}=\left\{[x y]_{G},[y z]_{G \backslash e},[z x]_{G \backslash e}\right\}$ is a geodesic triangle in $G$, there exists $\alpha^{\prime} \in[x y]_{G} \cup[x z]_{G \backslash e}$ such that $d_{G}\left(\alpha_{2}, \alpha^{\prime}\right) \leq \delta(G)$. Hence, there exists $\alpha^{\prime \prime} \in\left([x y]_{G} \backslash e\right) \cup[x z]_{G}$ such that $d_{G \backslash e}\left(\alpha_{2}, \alpha^{\prime \prime}\right) \leq \delta(G)$ (if the geodesic joining $\alpha_{2}$ and $\alpha^{\prime}$ contains $e=[A, B]$, then $\left.A, B \in[x y]_{G}\right)$. If $\alpha^{\prime \prime} \in[x z]_{G \backslash e}$, then we obtain $d_{G \backslash e}\left(\alpha_{2}, \alpha^{\prime \prime}\right) \leq \delta(G)$. Assume now that $\alpha^{\prime \prime} \in[x y]_{G} \backslash e$. By Lemma 3.4 there exists $\beta_{2} \in[x y]_{G \backslash e}$ such that $d_{G \backslash e}\left(\alpha^{\prime \prime}, \beta_{2}\right) \leq 2 \delta(G)$, and we have $d_{G \backslash e}\left(\alpha_{2}, \beta_{2}\right) \leq 3 d(G)$.

Therefore, we obtain $\delta\left(T_{G \backslash e}\right) \leq 4 \delta(G)$ in the case $n=1$.

Case $n=2$. Without loss of generality we can assume that $[y z]_{G \backslash e}=[y z]_{G}$.

Let us consider $\alpha \in[x y]_{G \backslash e} \cup[x z]_{G \backslash e}$; without loss of generality we can assume that $\alpha \in[x y]_{G \backslash e}$ and that $d_{G}(x, A)<d_{G}(x, B)$. By Lemma 3.2, for any $\alpha \in[x y]_{G \backslash e}$ there exists $\alpha^{\prime} \in[x y]_{G} \backslash e$ such that $d_{G \backslash e}\left(\alpha, \alpha^{\prime}\right) \leq 2 \delta(G)$. If $\alpha^{\prime} \in[y B]_{G}$, then since $T^{\prime}=\left\{[y B]_{G},[B z]_{G},[z y]_{G \backslash e}\right\}$ is $\delta(G)$-thin in $G$ there exists $\beta_{0}^{\prime} \in[y z]_{G} \cup[B z]_{G}$ such that $d_{G}\left(\alpha^{\prime}, \beta_{0}^{\prime}\right) \leq \delta(G)$; hence, there exists $\beta^{\prime} \in[y z]_{G} \cup\left([x z]_{G} \backslash e\right)$ such that $d_{G \backslash e}\left(\alpha^{\prime}, \beta^{\prime}\right) \leq \delta(G)$, since if the geodesic joining $\alpha^{\prime}$ and $\beta_{0}^{\prime}$ contains $e$, then we can take $\alpha^{\prime} \in\{A, B\}$. Moreover, if $\beta^{\prime} \in[y z]_{G}$, then $d_{G \backslash e}\left(\alpha, \beta^{\prime}\right) \leq 3 \delta(G)$. If $\beta^{\prime} \in[x z]_{G} \backslash e$, then by Lemma 3.4, there exists $\beta \in[x z]_{G \backslash e}$ such that $d_{G \backslash e}\left(\beta^{\prime}, \beta\right) \leq 2 \delta(G)$. Hence, we have $d_{G \backslash e}(\alpha, \beta) \leq 5 \delta(G)$. If $\alpha^{\prime} \in[x A]_{G}$, then we also obtain $d_{G \backslash e}(\alpha, \beta) \leq 5 \delta(G)$ with a similar argument.

Consider now $\alpha \in[y z]_{G \backslash e}$; since $T^{\prime}=\left\{[y B]_{G},[B z]_{G},[z y]_{G \backslash e}\right\}$ is $\delta(G)$-thin in $G$, there exists $\alpha_{0}^{\prime} \in[y B]_{G} \cup[B z]_{G}$ such that $d_{G}\left(\alpha, \alpha_{0}^{\prime}\right) \leq \delta(G)$. Thus, there exists $\alpha^{\prime} \in\left([x y]_{G} \cup[x z]_{G}\right) \backslash e$ such that $d_{G \backslash e}\left(\alpha, \alpha^{\prime}\right) \leq \delta(G)$, since if the geodesic joining $\alpha$ and $\alpha_{0}^{\prime}$ contains $e$, then we can take $\alpha^{\prime} \in\{A, B\}$. Hence, without loss of generality we can suppose that $\alpha^{\prime} \in[x y]_{G} \backslash e$; then by Lemma 3.4 there exists $\beta \in[x y]_{G \backslash e}$ such that $d_{G \backslash e}\left(\alpha^{\prime}, \beta\right) \leq 2 \delta(G)$. Therefore, we have $d_{G \backslash e}(\alpha, \beta) \leq 3 \delta(G)$.

Finally, we obtain $\delta\left(T_{G \backslash e}\right) \leq 5 \delta(G)$ in this case.

We will prove now a kind of converse of Theorem 3.9. First of all, note that it is not possible to have the inequality $\delta(G) \leq c \delta(G \backslash e)$ for some fixed constant $c$, since if $G$ is the cycle graph with $n$ vertices and edges with length 1 , and $e$ is any edge of $G$, then $\delta(G)=n / 4$ and $\delta(G \backslash e)=0$.

We prove first some previous results.

Lemma 3.10. Let $G$ be any graph and $e \in E(G)$ with $G \backslash e$ connected. Let $T_{G}$ be a geodesic triangle in $G$ contained in $G \backslash e$. Then $T_{G}$ is $\delta(G \backslash e)$-thin in $G$, i.e.,

$$
\delta\left(T_{G}\right) \leq \delta(G \backslash e)
$$


Proof. This result is straightforward since $T_{G}$ is a geodesic triangle in $G \backslash e$ also, and $d_{G}(x, y) \leq d_{G \backslash e}(x, y)$ for every $x, y \in G \backslash e$.

Lemma 3.11. Let $G$ be any graph and $e=[A, B] \in E(G)$ with $G \backslash$ e connected. For all $x, y \in G \backslash e$, if $\Gamma_{G}=[x y]_{G}$ is a geodesic in $G$ containing e and $\Gamma_{G \backslash e}=[x y]_{G \backslash e}$ is a geodesic in $G \backslash e$, then

$$
\forall u \in \Gamma_{G \backslash e}, \exists u^{\prime} \in \Gamma_{G} \backslash e: d_{G}\left(u, u^{\prime}\right) \leq 2 \delta(G \backslash e)+\frac{1}{2} d_{G \backslash e}(A, B) .
$$

Proof. Without loss of generality we can assume that $G \backslash e$ is hyperbolic, since otherwise the inequality is direct. We can assume also that $\Gamma_{G}=[x y]_{G}=[x A] \cup e \cup[B y]$. Let us consider the geodesic quadrilateral $P_{4}=\left\{[x y]_{G \backslash e},[x A],[A B]_{G \backslash e},[B y]\right\}$ in $G \backslash e$. Since $P_{4}$ is $2 \delta(G \backslash e)$-thin in $G \backslash e$, then

$$
\forall u \in \Gamma_{G \backslash e}, \quad d_{G \backslash e}\left(u,[x A] \cup[A B]_{G \backslash e} \cup[B y]\right) \leq 2 \delta(G \backslash e),
$$

and inequality (3.7) follows.

Lemma 3.12. Let $G$ be any graph and $e=[A, B] \in E(G)$ with $G \backslash e$ connected. For all $x, y \in G \backslash e$, if $\Gamma_{G}=[x y]_{G}$ is a geodesic in $G$ containing e and $\Gamma_{G \backslash e}=[x y]_{G \backslash e}$ is a geodesic in $G \backslash e$, then

$$
\forall u^{\prime} \in \Gamma_{G}, \exists u \in \Gamma_{G \backslash e}: d_{G}\left(u^{\prime}, u\right) \leq 5 \delta(G \backslash e)+d_{G \backslash e}(A, B) .
$$

Proof. Without loss of generality we can assume that $G \backslash e$ is hyperbolic, since otherwise the inequality is direct. We can assume also that $\Gamma_{G}=[x y]_{G}=[x A] \cup e \cup[B y]$. Denoted by $P$ the middle point of $[A B]_{G \backslash e}$. Note that the condition $e \subseteq \Gamma_{G}=[x y]_{G}$, implies $d_{G \backslash e}(A, B) \geq L(e)$.

Note also that

$$
\forall u^{\prime} \in \Gamma_{G}, \exists u^{*} \in[x A] \cup[B y] \quad: \quad d_{G}\left(u, u^{*}\right) \leq \frac{1}{2} L(e) .
$$

Without loss of generality we can assume that $u^{*} \in[x A]$. Since $T=\left\{[x A],[A P]_{G \backslash e}\right.$, $\left.[x P]_{G \backslash e}\right\}$ is a geodesic triangle in $G \backslash e$, there exists $\alpha \in[A P]_{G \backslash e} \cup[x P]_{G \backslash e}$ such that $d_{G}\left(u^{*}, \alpha\right) \leq d_{G \backslash e}\left(u^{*}, \alpha\right) \leq \delta(G \backslash e)$, and so

$$
\forall u^{*} \in[x A], \exists \beta \in[x P]_{G \backslash e} \quad: \quad d_{G}\left(u^{*}, \beta\right) \leq \delta(G \backslash e)+\frac{1}{2} d_{G \backslash e}(A, B) .
$$

Now, $T=\left\{[x y]_{G \backslash e},[x P]_{G \backslash e},[P y]_{G \backslash e}\right\}$ is a geodesic triangle in $G \backslash e$ and $T$ is $4 \delta(G \backslash e)$-fine by Theorem 2.2. Let us denote by $r, s$ and $t$ the internal points in the geodesics $[x y]_{G \backslash e}$, $[x P]_{G \backslash e}$ and $[P y]_{G \backslash e}$, respectively. Since $L\left([s P]_{G \backslash e}\right)=L\left([P t]_{G \backslash e}\right)=\frac{1}{2}\left(L\left([x P]_{G \backslash e}\right)+\right.$ $\left.L\left([P y]_{G \backslash e}\right)-L\left([x y]_{G \backslash e}\right)\right)$, we have

$$
\begin{gathered}
\forall \beta \in[x P]_{G \backslash e} \cup[P y]_{G \backslash e}, \exists u \in[x y]_{G \backslash e}: \\
d_{G}(\beta, u) \leq 4 \delta(G \backslash e)+\frac{1}{2}\left(L\left([x P]_{G \backslash e}\right)+L\left([P y]_{G \backslash e}\right)-L\left([x y]_{G \backslash e}\right)\right) .
\end{gathered}
$$


The triangle inequality gives

$$
\begin{aligned}
L\left([x P]_{G \backslash e}\right)+L\left([P y]_{G \backslash e}\right) & \leq L\left([x A]_{G \backslash e}\right)+L\left([A P]_{G \backslash e}\right)+L\left([P B]_{G \backslash e}\right)+L\left([B y]_{G \backslash e}\right) \\
& =L\left([x y]_{G}\right)+d_{G \backslash e}(A, B)-L(e) .
\end{aligned}
$$

Since $L\left([x y]_{G \backslash e}\right) \geq L\left([x y]_{G}\right)$, we deduce

$$
\frac{1}{2}\left(L\left([x P]_{G \backslash e}\right)+L\left([P y]_{G \backslash e}\right)-L\left([x y]_{G \backslash e}\right)\right) \leq \frac{1}{2}\left(d_{G \backslash e}(A, B)-L(e)\right) .
$$

Finally, if we consider the path $\left[u^{\prime} u^{*}\right] \cup\left[u^{*} \beta\right] \cup[\beta u]$, then we obtain

$$
\begin{aligned}
d_{G}\left(u^{\prime}, u\right) & \leq \frac{1}{2} L(e)+\delta(G \backslash e)+\frac{1}{2} d_{G \backslash e}(A, B)+4 \delta(G \backslash e)+\frac{1}{2}\left(d_{G \backslash e}(A, B)-L(e)\right) \\
& =5 \delta(G \backslash e)+d_{G \backslash e}(A, B) .
\end{aligned}
$$

Lemma 3.13. Let $G$ be any graph and $e=[A, B] \in E(G)$ with $G \backslash$ e connected. Let $T_{G}=$ $\left\{[x y]_{G},[y z]_{G},[z x]_{G}\right\}$ be a geodesic triangle in $G$, such that $e \subseteq[x y]_{G}$ and $[y z]_{G},[z x]_{G} \subset$ $G \backslash e$. Then

$$
\delta\left(T_{G}\right) \leq 6 \delta(G \backslash e)+d_{G \backslash e}(A, B) .
$$

Proof. Without loss of generality we can assume that $G \backslash e$ is hyperbolic, since otherwise the inequality is direct. Let $[x y]_{G \backslash e}$ be a geodesic in $G \backslash e$; then $T=\left\{[x y]_{G \backslash e},[y z]_{G},[z x]_{G}\right\}$ is a geodesic triangle in $G \backslash e$. Hence, for any $\alpha \in[y z]_{G}$ we have

$$
d_{G}\left(\alpha,[z x]_{G} \cup[x y]_{G \backslash e}\right) \leq d_{G \backslash e}\left(\alpha,[z x]_{G} \cup[x y]_{G \backslash e}\right) \leq \delta(G \backslash e) .
$$

By Lemma 3.11, for any $\beta \in[x y]_{G \backslash e}$, we have $d_{G}\left(\beta,[x y]_{G}\right) \leq 2 \delta(G \backslash e)+\frac{1}{2} d_{G \backslash e}(A, B)$. Then we obtain

$$
d_{G}\left(\alpha,[z x]_{G} \cup[x y]_{G}\right) \leq 3 \delta(G \backslash e)+\frac{1}{2} d_{G \backslash e}(A, B) .
$$

If $\alpha \in[z x]_{G}$, then the same argument gives the last inequality.

By Lemma 3.12, for any $\alpha \in[x y]_{G}$, there exists $\beta \in[x y]_{G \backslash e}$ such that $d_{G}(\alpha, \beta) \leq$ $5 \delta(G \backslash e)+d_{G \backslash e}(A, B)$. If we consider again the geodesic triangle $T$ in $G \backslash e$, then we have

$$
d_{G}\left(\beta,[y z]_{G} \cup[z x]_{G}\right) \leq d_{G \backslash e}\left(\beta,[y z]_{G} \cup[z x]_{G}\right) \leq \delta(G \backslash e),
$$

Therefore, for any $\alpha \in[x y]_{G}$, we obtain

$$
d_{G}\left(\alpha,[y z]_{G} \cup[z x]_{G}\right) \leq 6 \delta(G \backslash e)+d_{G \backslash e}(A, B) .
$$

Lemma 3.14. Let $G$ be any graph and $e=[A, B] \in E(G)$ with $G \backslash e$ connected. Let $T_{G}=\left\{[x y]_{G},[y z]_{G},[z x]_{G}\right\}$ be a geodesic triangle in $G$, such that $\{x, y, z\} \cap e \neq \varnothing$. Then

$$
\delta\left(T_{G}\right) \leq \max \left\{2 \delta(G \backslash e)+d_{G \backslash e}(A, B), L(e)\right\} .
$$


Proof. Without loss of generality we can assume that $G \backslash e$ is hyperbolic, since otherwise the inequality is direct. If every vertex of $T_{G}$ belongs to $e$, then we have $T_{G} \subseteq e \cup[A B]_{G \backslash e}$; hence, $\delta\left(T_{G}\right) \leq \frac{1}{4} L\left(T_{G}\right)=\frac{1}{4}\left(L(e)+d_{G \backslash e}(A, B)\right)$.

Assume now that there are exactly two vertices of $T_{G}$ in $e$; without loss of generality we can assume that $x, y \in e, z \notin e, A \in[x z]_{G}$ and $B \in[y z]_{G}$. In order to bound $\delta\left(T_{G}\right)$, let us choose any $\alpha \in T_{G}$. If $\alpha \in[x y]_{G}$, then we have $d_{G}\left(\alpha,[x z]_{G} \cup[y z]_{G}\right)=d_{G}(\alpha,\{x, y\}) \leq L(e)$. If $\alpha \in[x z]_{G} \cup[y z]_{G}$, then without loss of generality we can assume that $\alpha \in[x z]_{G}$. If $\alpha \in[x A]_{G} \subset[x z]_{G}$, then we have $d_{G}\left(\alpha,[x y]_{G} \cup[y z]_{G}\right) \leq d_{G}(\alpha, x) \leq L(e)$. If $\alpha \in[A z]_{G} \subset$ $[x z]_{G}$, then let us consider the geodesic triangle $T^{*}=\left\{[A z]_{G},[z B]_{G},[A B]_{G \backslash e}\right\}$ in $G \backslash e$; then there exists $\beta \in[B z]_{G} \cup[A B]_{G \backslash e}$ such that $d_{G}(\alpha, \beta) \leq d_{G \backslash e}(\alpha, \beta) \leq \delta(G \backslash e)$, and we obtain $d_{G}\left(\alpha,[y z]_{G} \cup[x y]_{G}\right) \leq \delta(G \backslash e)+d_{G \backslash e}(A, B)$. Hence,

$$
\delta\left(T_{G}\right) \leq \max \left\{\delta(G \backslash e)+d_{\backslash e}(A, B), L(e)\right\} .
$$

Finally, assume that there is exactly one vertex of $T_{G}$ in $e$; without loss of generality we can assume that $x \in e, z, y \notin e, A \in[x y]_{G}$ and $B \in[x z]_{G}$. In order to bound $\delta\left(T_{G}\right)$, let us choose any $\alpha \in T_{G}$. If $\alpha \in[y z]_{G}$, then $T_{4}^{*}=\left\{[A y]_{G},[y z]_{G},[z B]_{G},[A B]_{G \backslash e}\right\}$ is a geodesic quadrilateral in $G \backslash e$ and there exists $\beta \in[y A]_{G} \cup[A B]_{G \backslash e} \cup[B z]_{G}$ such that $d_{G}(\alpha, \beta) \leq d_{G \backslash e}(\alpha, \beta) \leq 2 \delta(G \backslash e)$; hence, we obtain $d_{G}\left(\alpha,[y x]_{G} \cup[x z]_{G}\right) \leq 2 \delta(G \backslash e)+$ $\frac{1}{2} d_{G \backslash e}(A, B)$. If $\alpha \in[x y]_{G} \cup[x z]_{G}$, then without loss of generality we can assume that $\alpha \in[x y]_{G}$. If $\alpha \in[x A]_{G} \subset[x y]_{G}$, then we have $d_{G}\left(\alpha,[x z]_{G} \cup[y z]_{G}\right) \leq d_{G}(\alpha, x) \leq L(e)$. If $\alpha \in[A y]_{G} \subset[x y]_{G}$, then let us consider again the geodesic quadrilateral $T_{4}^{*}$; hence, there exists $\beta \in[A B]_{G \backslash e} \cup[B z]_{G} \cup[z y]_{G}$ such that $d_{G}(\alpha, \beta) \leq d_{G \backslash e}(\alpha, \beta) \leq 2 \delta(G \backslash e)$, and we obtain $d_{G}\left(\alpha,[y z]_{G} \cup[z x]_{G}\right) \leq 2 \delta(G \backslash e)+d_{G \backslash e}(A, B)$. Hence,

$$
\delta\left(T_{G}\right) \leq \max \left\{2 \delta(G \backslash e)+d_{G \backslash e}(A, B), L(e)\right\} .
$$

Finally, we can prove a kind of converse of Theorem 3.9.

Theorem 3.15. Let $G$ be any graph and $e=[A, B] \in E(G)$ with $G \backslash e$ connected. Then

$$
\max \left\{\frac{1}{5} \delta(G \backslash e), \frac{1}{4} d_{G \backslash e}(A, B), \frac{1}{4} L(e)\right\} \leq \delta(G) \leq \max \left\{6 \delta(G \backslash e)+d_{G \backslash e}(A, B), L(e)\right\} .
$$

Proof. Theorem 3.9 gives $\delta(G \backslash e) / 5 \leq \delta(G)$. If $d_{G \backslash e}(A, B) \leq L(e)$, then let $C_{1}$ be the midpoint of $e$ and $w_{1}$ the midpoint of $\left[A C_{1}\right]_{G}$; since $T_{1}=\left\{A, B, C_{1}\right\}$ is a geodesic triangle in $G$, we have $\delta(G) \geq \delta\left(T_{1}\right) \geq d_{G}\left(w_{1},[A B]_{G} \cup\left[B C_{1}\right]_{G}\right)=L(e) / 4$. If $d_{G \backslash e}(A, B) \geq L(e)$, then let $C_{2}$ be the midpoint of a geodesic $[A B]_{G \backslash e}$ and $w_{2}$ the midpoint of $\left[A C_{2}\right]_{G \backslash e} \subset[A B]_{G \backslash e}$ (note that $\left[A C_{2}\right]_{G \backslash e}$ is a geodesic in $G$ also); since $T_{2}=\left\{A, B, C_{2}\right\}$ is a geodesic triangle in $G$, we have $\delta(G) \geq \delta\left(T_{2}\right) \geq d_{G}\left(w_{2}, e \cup\left[B C_{2}\right]_{G}\right)=d_{G \backslash e}(A, B) / 4$. These facts prove the lower bound for $\delta(G)$.

In order to prove the second inequality, let us consider a geodesic triangle $T_{G}$ in $G$. By Lemma 3.14 we can assume that every vertex of $T_{G}$ is contained in $G \backslash e$. By Lemma 
3.6 at most two geodesics sides of $T_{G}$ contain $e$. If $T_{G} \subseteq G \backslash e$, then Lemma 3.10 gives the result. If just one geodesic side of $T_{G}$ contains $e$, then it suffices to apply Lemma 3.13. If two geodesics sides of $T_{G}$ contain $e$, then we can split $T_{G}$ in the union of $e$, a geodesic bigon in $G \backslash e$ and a geodesic triangle in $G \backslash e$, and Lemma 3.10 finishes the proof.

We have the following direct consequences.

Corollary 3.16. Let $G$ be any graph and $e=[A, B] \in E(G)$ with $G \backslash$ e connected. Then

$$
\frac{1}{5} \max \left\{\delta(G \backslash e), d_{G \backslash e}(A, B), L(e)\right\} \leq \delta(G) \leq 12 \max \left\{\delta(G \backslash e), d_{G \backslash e}(A, B), L(e)\right\} .
$$

Corollary 3.17. Let $G$ be any graph and $e=[A, B] \in E(G)$ such that $G \backslash e$ is connected and $L(e) \leq d_{G \backslash e}(A, B)$. Then

$$
\delta(G) \leq 6 \delta(G \backslash e)+d_{G \backslash e}(A, B) .
$$

\section{Hyperbolic S-graphs}

Using the previous results, we prove in this section that local hyperbolicity guarantees the hyperbolicity of any graph, in a quantitative way. In order to do that we need to introduce the concept of S-graph.

Definition 4.1. Let us consider a graph $G_{0}$ with $E\left(G_{0}\right)=\left\{\left[a_{n}, b_{n}\right]\right\}_{n \geq 1}$, and a family of graphs $\left\{G_{n}\right\}_{n \geq 1}$ such that for all $n \geq 1$ there exist $a_{n}^{\prime}, b_{n}^{\prime} \in V\left(G_{n}\right)$ such that $d_{G_{n}}\left(a_{n}^{\prime}, b_{n}^{\prime}\right)=$ $L_{G_{0}}\left(\left[a_{n}, b_{n}\right]\right)$. We define the S-graph $G$ associated to $\left\{G_{n}\right\}_{n \geq 0}$ as follows; we replace each edge $\left[a_{n}, b_{n}\right] \in E\left(G_{0}\right)$ by the whole graph $G_{n}$ in the following way: $a_{n}$ and $b_{n}$ are substituted, respectively, by $a_{n}^{\prime}$ and $b_{n}^{\prime}$, for each $n \geq 1$.

A very simple example of S-graph is the following: Let $G$ be any graph with at least two connection vertices $v, w$ (recall that a connection vertex is a vertex whose removal renders $G$ disconnected). We denote by $G_{1}, G_{2}, G_{3}$, the closures in $G$ of the connected components of the metric graph $G$ minus the points $\{v, w\}$. Without loss of generality we can assume that $v \in G_{1}, v, w \in G_{2}$ and $w \in G_{3}$. If $\alpha \neq v$ is a vertex of $G_{1}$ and $\beta \neq w$ is a vertex of $G_{3}$, we define $G_{0}$ as the graph with $V\left(G_{0}\right)=\{\alpha, v, w, \beta\}, E\left(G_{0}\right)=\{[\alpha, v],[v, w],[w, \beta]\}$ and $L([\alpha, v])=d_{G}(\alpha, v), L([v, w])=d_{G}(v, w), L([w, \beta])=d_{G}(w, \beta)$. Then $G$ is the S-graph associated to $\left\{G_{0}, G_{1}, G_{2}, G_{3}\right\}$.

The previous example shows that we can view the graphs as S-graphs.

As usual, by cycle in a graph we mean a simple closed curve, i.e., a path with different vertices, unless the last vertex, which is equal to the first one.

In [31, Lemma 2.1] or [3, Corollary 4] we found the following result. Recall that a triangle is a cycle if and only if it has no self-intersection.

Lemma 4.2. In any graph $G$,

$$
\delta(G)=\sup \{\delta(T): T \text { is a geodesic triangle that is a cycle }\} .
$$


Theorem 4.3. Let $G$ be the $S$-graph associated to $\left\{G_{n}\right\}_{n \geq 0}$. Then, $G$ is hyperbolic if and only if $\left\{G_{n}\right\}_{n \geq 0}$ are hyperbolic with the same hyperbolicity constant. Furthermore,

$$
\frac{1}{5} \sup _{n \geq 0} \delta\left(G_{n}\right) \leq \delta(G) \leq 11 \sup _{n \geq 0} \delta\left(G_{n}\right) .
$$

Proof. Assume first that $G$ is hyperbolic. For each $n \geq 1$, let us denote by $\left[a_{n}^{\prime} b_{n}^{\prime}\right]_{G_{n}}$ a geodesic in $G_{n}$, and define $G^{*}$ as the subgraph of $G$ given by $G^{*}=\cup_{n \geq 1}\left[a_{n}^{\prime} b_{n}^{\prime}\right]_{G_{n}}$. Note that $G^{*}$ and $G_{0}$ are isometric. We have that $G^{*}$ is an isometric subgraph of $G$ and Lemma 3.8 gives $\delta\left(G_{0}\right)=\delta\left(G^{*}\right) \leq \delta(G)$. In what follows we identify $G^{*}$ and $G_{0}$. For each $n \geq 1$, if $G \backslash G_{n}$ is connected, let us consider a geodesic $\alpha_{n}$ in $G \backslash G_{n}$ joining $a_{n}^{\prime}$ and $b_{n}^{\prime}$; if $G \backslash G_{n}$ is not connected, we define $\alpha_{n}=\varnothing$; then $G_{n} \cup \alpha_{n}$ is an isometric subgraph of $G$. Therefore, by Theorem 3.9 and Lemma 3.8, we have that $\delta\left(G_{n}\right) \leq 5 \delta\left(G_{n} \cup \alpha_{n}\right) \leq 5 \delta(G)$. Hence, $G_{n}$ is $5 \delta(G)$-hyperbolic for every $n \geq 0$.

Assume now that $G_{n}$ is $\delta$-hyperbolic for every $n \geq 0$. Let us consider any fixed geodesic triangle $T=\{x, y, z\}$ in $G$; by Lemma 4.2 we can assume that $T$ is a cycle.

If $x, y, z$ belong to different subgraphs $G_{s}, G_{r}, G_{t}$, respectively, then let us consider the three geodesic triangles $T_{s}=\left\{x, a_{s}^{\prime}, b_{s}^{\prime}\right\}, T_{r}=\left\{y, a_{r}^{\prime}, b_{r}^{\prime}\right\}$ and $T_{t}=\left\{z, a_{t}^{\prime}, b_{t}^{\prime}\right\}$ in $G_{s}, G_{r}$ and $G_{t}$, respectively, and their tripods (see Definition 2.1). Let $P_{x}$ (respectively, $P_{y}, P_{z}$ ) be the internal point of $T_{s}$ in $\left[a_{s}^{\prime} b_{s}^{\prime}\right]$ (respectively, $T_{r}$ in $\left[a_{r}^{\prime} b_{r}^{\prime}\right], T_{t}$ in $\left[a_{t}^{\prime} b_{t}^{\prime}\right]$ ).

Since $T$ is a cycle and we are identifying $G^{*}$ and $G_{0}$, without loss of generality we can assume that

$$
\begin{gathered}
{[x y]=\left[x b_{s}\right]_{G_{s}} \cup\left[b_{s} a_{r}\right]_{G_{0}} \cup\left[a_{r} y\right]_{G_{r}},} \\
{[y z]=\left[y b_{r}\right]_{G_{r}} \cup\left[b_{r} a_{t}\right]_{G_{0}} \cup\left[a_{t} z\right]_{G_{t}}}
\end{gathered}
$$

and

$$
[z x]=\left[z b_{t}\right]_{G_{t}} \cup\left[b_{t} a_{s}\right]_{G_{0}} \cup\left[a_{s} x\right]_{G_{s}} .
$$

We are going to prove that $\left[P_{x} b_{s}\right]_{G_{0}} \cup\left[b_{s} a_{r}\right]_{G_{0}} \cup\left[a_{r} P_{y}\right]_{G_{0}}$ is a geodesic in $G_{0}$. Let $\left[P_{x} P_{y}\right]_{G_{0}}=\left[P_{x} c_{s}\right]_{G_{0}} \cup\left[c_{s} c_{r}\right]_{G_{0}} \cup\left[c_{r} P_{y}\right]_{G_{0}}$ be a geodesic in $G_{0}$ joining $P_{x}$ and $P_{y}$, where $c_{s} \in\left\{a_{s}, b_{s}\right\}$ and $c_{r} \in\left\{a_{r}, b_{r}\right\}$. Seeking for a contradiction, assume that $L\left(\left[P_{x} P_{y}\right]_{G_{0}}\right)<$ $L\left(\left[P_{x} b_{s}\right]_{G_{0}}\right)+L\left(\left[b_{s} a_{r}\right]_{G_{0}}\right)+L\left(\left[a_{r} P_{y}\right]_{G_{0}}\right)$. Denote by $P_{a_{s}}$ and $P_{b_{s}}$ the internal points of $T_{s}$ in $\left[x a_{s}^{\prime}\right]_{G_{s}}$ and $\left[x b_{s}^{\prime}\right]_{G_{s}}$, respectively; denote by $P_{a_{r}}$ and $P_{b_{r}}$ the internal points of $T_{r}$ in $\left[y a_{r}^{\prime}\right]_{G_{s}}$ and $\left[y b_{r}^{\prime}\right]_{G_{s}}$, respectively. Then

$$
\begin{aligned}
L\left(\left[P_{x} c_{s}\right]_{G_{s}}\right) & +L\left(\left[c_{s} c_{r}\right]_{G_{0}}\right)+L\left(\left[c_{r} P_{y}\right]_{G_{r}}\right)<L\left(\left[P_{x} b_{s}\right]_{G_{s}}\right)+L\left(\left[b_{s} a_{r}\right]_{G_{0}}\right)+L\left(\left[a_{r} P_{y}\right]_{G_{r}}\right), \\
L\left(\left[P_{c_{s}} c_{s}\right]_{G_{s}}\right) & +L\left(\left[c_{s} c_{r}\right]_{G_{0}}\right)+L\left(\left[c_{r} P_{c_{r}}\right]_{G_{r}}\right)<L\left(\left[P_{b_{s}} b_{s}\right]_{G_{s}}\right)+L\left(\left[b_{s} a_{r}\right]_{G_{0}}\right)+L\left(\left[a_{r} P_{a_{r}}\right]_{G_{r}}\right), \\
d_{G}(x, y) & \leq L\left(\left[x P_{c_{s}}\right]_{G_{s}}\right)+L\left(\left[P_{c_{s}} c_{s}\right]_{G_{s}}\right)+L\left(\left[c_{s} c_{r}\right]_{G_{0}}\right)+L\left(\left[c_{r} P_{c_{r}}\right]_{G_{r}}\right)+L\left(\left[P_{c_{r}} y\right]_{G_{r}}\right) \\
& <L\left(\left[x P_{b_{s}}\right]_{G_{s}}\right)+L\left(\left[P_{b_{s}} b_{s}\right]_{G_{s}}\right)+L\left(\left[b_{s} a_{r}\right]_{G_{0}}\right)+L\left(\left[a_{r} P_{a_{r}}\right]_{G_{r}}\right)+L\left(\left[P_{a_{r}} y\right]_{G_{r}}\right) \\
& =L([x y])=d_{G}(x, y),
\end{aligned}
$$

which is a contradiction. Then, we have that $\left[P_{x} b_{s}\right]_{G_{0}} \cup\left[b_{s} a_{r}\right]_{G_{0}} \cup\left[a_{r} P_{y}\right]_{G_{0}}$ is a geodesic in $G_{0}$ joining $P_{x}$ and $P_{y}$. A similar argument proves that $\left[P_{y} b_{r}\right]_{G_{0}} \cup\left[b_{r} a_{t}\right]_{G_{0}} \cup\left[a_{t} P_{z}\right]_{G_{0}}$ and $\left[P_{z} b_{t}\right]_{G_{0}} \cup\left[b_{t} a_{s}\right]_{G_{0}} \cup\left[a_{s} P_{x}\right]_{G_{0}}$ are also geodesics in $G_{0}$. Now, let us consider the geodesic triangle $T_{0}=\left\{P_{x}, P_{y}, P_{z}\right\}$ in $G_{0}$ with these geodesics. 
Let us consider any $\alpha \in T$. Without loss of generality we can assume that $\alpha \in[x y]$. If $\alpha \in\left[x b_{s}\right]_{G_{s}}$, then since $T_{s}$ is $\delta$-thin there exists $\alpha^{\prime} \in\left[x a_{s}\right]_{G_{s}} \cup\left[a_{s} b_{s}\right]_{G_{s}}$ such that $d_{G_{s}}\left(\alpha, \alpha^{\prime}\right) \leq \delta$. If $\alpha^{\prime} \in\left[x a_{s}\right]_{G_{s}}$, then $\alpha^{\prime} \in[x z]$. Assume now that $\alpha^{\prime} \in\left[a_{s} b_{s}\right]_{G_{s}}$. If $\alpha^{\prime} \in$ $\left[a_{s} P_{x}\right]_{G_{s}}$, then there exists $\beta \in\left[x a_{s}\right]_{G_{s}} \subset[x z]$ such that $d_{G_{s}}\left(\alpha^{\prime}, \beta\right) \leq 4 \delta$ and $d_{G}(\alpha, \beta) \leq 5 \delta$. If $\alpha^{\prime} \in\left[P_{x} b_{s}\right]_{G_{s}} \subset\left[P_{x} P_{y}\right]_{G_{0}}$ since $T_{0}$ is $\delta$-thin, there exists $\beta^{\prime} \in\left[P_{y} P_{z}\right]_{G_{0}} \cup\left[P_{z} P_{x}\right]_{G_{0}}$ such that $d_{G_{0}}\left(\alpha^{\prime}, \beta^{\prime}\right) \leq \delta$. Then, $\beta^{\prime}$ belongs to $\left[b_{r} a_{t}\right]_{G_{0}} \cup\left[b_{t} a_{s}\right]_{G_{0}} \subset[y z]_{G} \cup[z x]_{G}$ or to one of the subgraphs $T_{s}, T_{r}$ or $T_{t}$ (if $\beta^{\prime}$ belongs to $\left[P_{y} b_{r}\right]_{G_{r}},\left[a_{t} P_{z}\right]_{G_{t}},\left[P_{z} b_{t}\right]_{G_{t}},\left[a_{s} P_{x}\right]_{G_{s}}$ ) and there exists $\beta \in[y z]_{G} \cup[z x]_{G}$ such that $d_{G}\left(\beta^{\prime}, \beta\right) \leq 4 \delta$. Then, we obtain $d_{G}(\alpha, \beta) \leq$ $d_{G_{s}}\left(\alpha, \alpha^{\prime}\right)+d_{G_{0}}\left(\alpha^{\prime}, \beta^{\prime}\right)+d_{G}\left(\beta^{\prime}, \beta\right) \leq 6 \delta$. Note that, by symmetry, if $\alpha \in\left[a_{r} y\right]_{G_{r}}$ we have the same result. If $\alpha \in\left[b_{s} a_{r}\right]_{G_{0}}$, then since $T_{0}$ is $\delta$-thin there exists $\beta^{\prime} \in\left[P_{y} P_{z}\right]_{G_{0}} \cup\left[P_{z} P_{x}\right]_{G_{0}}$ such that $d_{G_{0}}\left(\alpha, \beta^{\prime}\right) \leq \delta$. Then, $\beta^{\prime}$ belongs to $\left[b_{r} a_{t}\right]_{G_{0}} \cup\left[b_{t} a_{s}\right]_{G_{0}} \subset[y z]_{G} \cup[z x]_{G}$ or to one of the subgraphs $T_{s}, T_{r}$ or $T_{t}$ (if $\beta^{\prime}$ belongs to $\left[P_{y} b_{r}\right]_{G_{r}},\left[a_{t} P_{z}\right]_{G_{t}},\left[P_{z} b_{t}\right]_{G_{t}},\left[a_{s} P_{x}\right]_{G_{s}}$ ) and there exists $\beta \in[y z]_{G} \cup[z x]_{G}$ such that $d_{G}\left(\beta^{\prime}, \beta\right) \leq 4 \delta$. Then, we obtain $d_{G}(\alpha, \beta) \leq$ $d_{G_{0}}\left(\alpha, \beta^{\prime}\right)+d_{G}\left(\beta^{\prime}, \beta\right) \leq 5 \delta$. Consequently, if $x, y, z$ belong to different subgraphs, then

$$
\delta(T) \leq 6 \delta
$$

If $x, y$ belong to the same subgraph $G_{s}$ and $z \in G_{r}$ with $s \neq r$, then consider two geodesic polygons $F_{s}=\left\{x, y, a_{s}, b_{s}\right\}$ and $T_{r}=\left\{z, a_{r}, b_{r}\right\}$ in $G_{s}$ and $G_{r}$, respectively. Consider the tripod of $T_{r}$ and a quatripod of $F_{s}$ respectively, into the definition of fine. Let $P_{x}^{\prime}, P_{y}^{\prime}, P_{z}^{\prime}$ be the vertices with degree 3 in the quatripod and the tripod, respectively; let $P_{z}$ be the point in $\left[a_{r} b_{r}\right]$ related with $P_{z}^{\prime}$ (the internal point), and $P_{x}, P_{y} \in\left[a_{s} b_{s}\right]$ related with $P_{x}^{\prime}, P_{y}^{\prime}$ (note that it is possible to have $P_{x}=P_{y}$, in particular, if $P_{x}^{\prime}$ or $P_{y}^{\prime}$ is neighbor of the two vertices corresponding to $a_{s}$ and $b_{s}$ ).

Without loss of generality we can assume that

$$
\begin{aligned}
& {[y z]=\left[y b_{s}\right]_{G_{s}} \cup\left[b_{s} a_{r}\right]_{G_{0}} \cup\left[a_{r} z\right]_{G_{r}},} \\
& {[x z]=\left[x a_{s}\right]_{G_{s}} \cup\left[a_{s} b_{r}\right]_{G_{0}} \cup\left[b_{r} z\right]_{G_{r}}}
\end{aligned}
$$

and

$$
\left[a_{s} b_{s}\right]_{G_{s}}=\left[a_{s} P_{x}\right]_{G_{s}} \cup\left[P_{x} P_{y}\right]_{G_{s}} \cup\left[P_{y} b_{s}\right]_{G_{s}} .
$$

As in the previous case, it is possible to check that $\left[P_{x} a_{s}\right]_{G_{0}} \cup\left[a_{s} b_{r}\right]_{G_{0}} \cup\left[b_{r} P_{z}\right]_{G_{0}}$, $\left[P_{z} a_{r}\right]_{G_{0}} \cup\left[a_{r} b_{s}\right]_{G_{0}} \cup\left[b_{s} P_{y}\right]_{G_{0}}$ and $\left[P_{x} P_{y}\right]_{G_{0}}$ are geodesics in $G_{0}$. Let us consider the geodesic triangle $T_{0}=\left\{P_{x}, P_{y}, P_{z}\right\}$ in $G_{0}$ with these geodesics.

Let us fix any $\alpha \in[x y]$; there exists $\alpha^{\prime} \in\left[x a_{s}\right]_{G_{s}} \cup\left[a_{s} b_{s}\right]_{G_{s}} \cup\left[b_{s} y\right]_{G_{s}}$ such that $d_{G_{s}}\left(\alpha, \alpha^{\prime}\right) \leq 2 \delta$. If $\alpha^{\prime} \in\left[x a_{s}\right]_{G_{s}} \cup\left[b_{s} y\right]_{G_{s}}$, then $\alpha^{\prime} \in[x z] \cup[z y]$. If $\alpha^{\prime} \in\left[a_{s} P_{x}\right]_{G_{s}} \cup\left[P_{y} b_{s}\right]_{G_{s}}$, then by definition of fine quatripod there exists $\beta^{\prime} \in\left[x a_{s}\right]_{G_{s}} \cup\left[b_{s} y\right]_{G_{s}} \subset[x z] \cup[z y]$ such that $d_{G_{s}}\left(\alpha^{\prime}, \beta^{\prime}\right) \leq 8 \delta$. If $P_{x} \neq P_{y}$ and $\alpha^{\prime} \in\left[P_{x} P_{y}\right]_{G_{s}}$, then since $T_{0}$ is $\delta$-thin there exists $\beta^{\prime} \in\left[P_{y} P_{z}\right]_{G_{0}} \cup\left[P_{z} P_{x}\right]_{G_{0}}$ such that $d_{G_{0}}\left(\alpha^{\prime}, \beta^{\prime}\right) \leq \delta$; then, $\beta^{\prime} \in\left[b_{s} a_{r}\right]_{G_{0}} \cup\left[b_{r} a_{s}\right]_{G_{0}} \subset$ $[y z]_{G} \cup[z x]_{G}$ or since $F_{s}$ is $8 \delta$-fine and $T_{r}$ is $4 \delta$-fine there exists $\beta \in[y z]_{G} \cup[z x]_{G}$ such that $d_{G_{i}}\left(\beta^{\prime}, \beta\right) \leq 8 \delta$ with $i \in\{r, s\}$. Therefore, we conclude $d_{G}(\alpha, \beta) \leq 11 \delta$.

Let us fix now any $\alpha \in[x z] \cup[y z]$; without loss of generality we can assume that $\alpha \in[y z]$. 
Assume first that $\alpha \in\left[y b_{s}\right]_{G_{s}}$; then since $F_{s}$ is $2 \delta$-thin there exists $\alpha^{\prime} \in[x y]_{G} \cup$ $\left[x a_{s}\right]_{G_{s}} \cup\left[a_{s} b_{s}\right]_{G_{s}}$ such that $d_{G_{s}}\left(\alpha, \alpha^{\prime}\right) \leq 2 \delta$. If $\alpha^{\prime} \in[x y] \cup\left[x a_{s}\right]_{G_{s}}$, then $\alpha^{\prime} \in[x y] \cup[x z]$. Assume now that $\alpha^{\prime} \in\left[a_{s} b_{s}\right]_{G_{s}}$. If $\alpha^{\prime} \in\left[a_{s} P_{x}\right]_{G_{s}}$, then since $F_{s}$ is $8 \delta$-fine there exists $\beta^{\prime} \in\left[x a_{s}\right]_{G_{s}} \subset[x z]$ such that $d_{G_{s}}\left(\alpha^{\prime}, \beta^{\prime}\right) \leq 8 \delta$ and $d_{G}\left(\alpha, \beta^{\prime}\right) \leq 10 \delta$. If $P_{x} \neq P_{y}$ and $\alpha^{\prime} \in\left[P_{x} P_{y}\right]_{G_{s}}$, then there exists $\beta^{\prime} \in[x y]_{G}$ such that $d_{G_{s}}\left(\alpha^{\prime}, \beta^{\prime}\right) \leq 8 \delta$ and $d_{G}\left(\alpha, \beta^{\prime}\right) \leq 10 \delta$. If $\alpha^{\prime} \in\left[P_{y} b_{s}\right]_{G_{s}} \subset\left[P_{y} P_{z}\right]_{G_{0}}$, then since $T_{0}$ is $\delta$-thin, there exists $\beta^{\prime} \in\left[P_{z} P_{x}\right]_{G_{0}} \cup\left[P_{x} P_{y}\right]_{G_{0}}$ such that $d_{G_{0}}\left(\alpha^{\prime}, \beta^{\prime}\right) \leq \delta$. If $\beta^{\prime} \in\left[P_{x} P_{y}\right]_{G_{s}}$, then since $F_{s}$ is $8 \delta$-fine there exists $\beta \in[x y]_{G}$ such that $d_{G_{s}}\left(\beta^{\prime}, \beta\right) \leq 8 \delta$ and $d_{G}(\alpha, \beta) \leq 11 \delta$. Assume that $\beta^{\prime} \in\left[P_{z} P_{x}\right]_{G_{0}}$; if $\beta^{\prime} \in$ $\left[P_{z} b_{r}\right]_{G_{r}} \cup\left[P_{x} a_{s}\right]_{G_{s}}$, then since $F_{s}$ is $8 \delta$-fine and $T_{r}$ is $4 \delta$-fine there exists $\beta \in[z x]$ such that $d_{G_{i}}\left(\beta^{\prime}, \beta\right) \leq 8 \delta$ with $i \in\{r, s\}$ and therefore $d_{G}(\alpha, \beta) \leq 11 \delta$; otherwise, $\beta^{\prime} \in\left[b_{r} a_{s}\right]_{G_{0}} \subset$ $[z x]$ and $d_{G}\left(\alpha, \beta^{\prime}\right) \leq 3 \delta$.

Assume that $\alpha \in\left[b_{s} a_{r}\right]_{G_{0}} \subset\left[P_{y} P_{z}\right]_{G_{0}}$. Since $T_{0}$ is $\delta$-thin there exists $\alpha^{\prime} \in\left[P_{z} P_{x}\right]_{G_{0}} \cup$ $\left[P_{x} P_{y}\right]_{G_{0}}$ such that $d_{G_{0}}\left(\alpha, \alpha^{\prime}\right) \leq \delta$; using the previous arguments for $\alpha^{\prime} \in\left[P_{y} b_{s}\right]_{G_{s}}$, we obtain that there exists $\beta \in[x y] \cup[x z]$ such that $d_{G}(\alpha, \beta) \leq 9 \delta$.

Assume that $\alpha \in\left[a_{r} z\right]_{G_{r}}$; then since $T_{r}$ is $\delta$-thin there exists $\alpha^{\prime} \in\left[z b_{r}\right]_{G_{r}} \cup\left[b_{r} a_{r}\right]_{G_{r}}$ such that $d_{G_{r}}\left(\alpha, \alpha^{\prime}\right) \leq \delta$. If $\alpha^{\prime} \in\left[z b_{r}\right]_{G_{r}}$, then $\alpha^{\prime} \in[z x]$. If $\alpha^{\prime} \in\left[b_{r} P_{z}\right]_{G_{r}}$, then since $T_{r}$ is $4 \delta$-fine there exists $\beta^{\prime} \in\left[z b_{r}\right]_{G_{r}} \subset[z x]$ such that $d_{G_{r}}\left(\alpha^{\prime}, \beta^{\prime}\right) \leq 4 \delta$ and $d_{G}\left(\alpha, \beta^{\prime}\right) \leq 5 \delta$. If $\alpha^{\prime} \in\left[P_{z} a_{r}\right]_{G_{r}}$, then since $T_{0}$ is $\delta$-thin there exists $\beta^{\prime} \in\left[P_{z} P_{x}\right]_{G_{0}} \cup\left[P_{x} P_{y}\right]_{G_{0}}$ such that $d_{G_{0}}\left(\alpha^{\prime}, \beta\right) \leq \delta$; using the previous arguments for $\alpha^{\prime} \in\left[P_{y} b_{s}\right]_{G_{s}}$, we obtain that there exists $\beta \in[x y] \cup[x z]$ such that $d_{G}(\alpha, \beta) \leq 10 \delta$.

Consequently, if $x, y$ belong to the same subgraph $G_{s}$ and $z \in G_{r}$ with $s \neq r$, then

$$
\delta(T) \leq 11 \delta .
$$

Finally, assume that $x, y, z$ belong to the same subgraph $G_{s}$. If $T$ is contained in $G_{s}$, then $\delta(T) \leq \delta\left(G_{s}\right) \leq \delta$. Assume that $T$ is not contained in $G_{s}$; then $e=\left[a_{s}, b_{s}\right] \in E\left(G_{0}\right)$, $G_{0} \backslash e$ is connected and $L(e) \leq d_{G_{s}}\left(a_{s}^{\prime}, b_{s}^{\prime}\right)$. Hence, $T$ is contained in $G_{s} \cup \alpha_{s}$, where $\alpha_{s}$ is a geodesic in $G_{0} \backslash e$ joining $a_{s}$ and $b_{s}$. Corollary 3.17 gives

$$
\delta(T) \leq \delta\left(G_{s} \cup \alpha_{s}\right) \leq 6 \delta\left(G_{s}\right)+d_{G_{s}}\left(a_{s}^{\prime}, b_{s}^{\prime}\right) \leq 6 \delta+d_{G_{s}}\left(a_{s}^{\prime}, b_{s}^{\prime}\right) .
$$

Note that $\left[a_{s}, b_{s}\right] \cup \alpha_{s}$ is an isometric cycle in $G_{0}$; therefore,

$$
\frac{1}{4} d_{G_{s}}\left(a_{s}^{\prime}, b_{s}^{\prime}\right)=\frac{1}{4} L\left(\left[a_{s}, b_{s}\right]\right) \leq \frac{1}{4} L\left(\left[a_{s}, b_{s}\right] \cup \alpha_{s}\right) \leq \delta\left(\left[a_{s}, b_{s}\right] \cup \alpha_{s}\right) \leq \delta\left(G_{0}\right) \leq \delta .
$$

Consequently, if $x, y, z$ belong to the same subgraph, then $\delta(T) \leq 10 \delta$.

Finally, we obtain that $G$ is hyperbolic with $\delta(G) \leq 11 \delta$.

\section{Acknowledgements}

We would like to thank the referee for a careful reading of the manuscript and for some helpful suggestions.

This work was partly supported by the Spanish Ministry of Science and Innovation through projects MTM2009-07800, MTM2008-02829-E and a grant from CONACYT (CONACYT-UAG I0110/62/10), México. 


\section{References}

[1] Bermudo, S., Rodríguez, J. M. and Sigarreta, J. M., Computing the hyperbolicity constant, Comput. Math. Appl. 62 (12) (2011), 4592-4595.

[2] Bermudo, S., Rodríguez, J. M., Sigarreta, J. M. and Vilaire, J.-M., Mathematical properties of Gromov hyperbolic graphs, AIP Conference Proceedings, Vol. 1281 (2010), 575-578.

[3] Bermudo, S., Rodríguez, J. M., Sigarreta, J. M. and Vilaire, J.-M., Gromov hyperbolic graphs. Submitted.

[4] Bermudo, S., Rodríguez, J. M., Sigarreta, J. M. and Tourís, E., Hyperbolicity and complement of graphs, Appl. Math. Letters 24 (2011), 1882-1887.

[5] Bowditch, B. H., Notes on Gromov's hyperobolicity criterion for path-metric spaces. Group theory from a geometrical viewpoint, Trieste, 1990 (ed. E. Ghys, A. Haefliger and A. Verjovsky; World Scientific, River Edge, NJ, 1991) 64-167.

[6] Brinkmann, G., Koolen J. and Moulton, V., On the hyperbolicity of chordal graphs, Ann. Comb. 5 (2001), 61-69.

[7] Carballosa, W., Portilla, A., Rodríguez, J. M. and Sigarreta, J. M., Gromov hyperbolicity of planar graphs and CW complexes. Submitted.

[8] Carballosa, W., Rodríguez, J. M., Sigarreta, J. M. and Villeta, M., On the hyperbolicity constant of line graphs, Electr. J. Comb. 18 (2011), \#P210.

[9] Chen, B., Yau, S.-T., Yeh, Y.-N., Graph homotopy and Graham homotopy, Discrete Math. 241 (2001), 153-170.

[10] Chepoi, V., Dragan, F. F., Estellon, B., Habib, M. and Vaxes Y., Notes on diameters, centers, and approximating trees of $\delta$-hyperbolic geodesic spaces and graphs, Electr. Notes Discrete Math. 31 (2008), 231-234.

[11] Frigerio, R. and Sisto, A., Characterizing hyperbolic spaces and real trees, Geom Dedicata 142 (2009), 139-149.

[12] Ghys, E. and de la Harpe, P., Sur les Groupes Hyperboliques d'après Mikhael Gromov. Progress in Mathematics 83, Birkhäuser Boston Inc., Boston, MA, 1990.

[13] Gromov, M., Hyperbolic groups, in "Essays in group theory". Edited by S. M. Gersten, M. S. R. I. Publ. 8. Springer, 1987, 75-263.

[14] Gromov, M. (with appendices by M. Katz, P. Pansu and S. Semmes), Metric Structures for Riemannian and Non-Riemannnian Spaces. Progress in Mathematics, vol. 152. Birkhäuser, 1999.

[15] Jonckheere, E. and Lohsoonthorn, P., A hyperbolic geometry approach to multipath routing, Proceedings of the 10th Mediterranean Conference on Control and Automation (MED 2002), Lisbon, Portugal, July 2002. FA5-1.

[16] Jonckheere, E. A., Controle du trafic sur les reseaux a geometrie hyperbolique-Une approche mathematique a la securite de l'acheminement de l'information, Journal Europeen de Systemes Automatises 37(2) (2003), 145-159. 
[17] Jonckheere, E. A. and Lohsoonthorn, P., Geometry of network security, American Control Conference ACC (2004), 111-151.

[18] Jonckheere, E. A., Lohsoonthorn, P. and Ariaesi, F, Upper bound on scaled Gromovhyperbolic delta, Applied Mathematics and Computation 192 (2007), 191-204.

[19] Jonckheere, E. A., Lohsoonthorn, P. and Bonahon, F., Scaled Gromov hyperbolic graphs, Journal of Graph Theory 2 (2007), 157-180.

[20] Koolen, J. H. and Moulton, V., Hyperbolic Bridged Graphs, Europ. J. Combinatorics 23 (2002), 683-699.

[21] Michel, J., Rodríguez, J. M., Sigarreta, J. M. and Villeta, M., Hyperbolicity and parameters of graphs, Ars Comb. Volume C (2011), 43-63.

[22] Michel, J., Rodríguez, J. M., Sigarreta, J. M. and Villeta, M., Gromov hyperbolicity in cartesian product graphs, Proc. Indian Acad. Sci. Math. Sci. 120 (2010), 1-17.

[23] Oshika, K., Discrete groups, AMS Bookstore, 2002.

[24] Pestana, D., Rodríguez, J. M., Sigarreta, J. M. and Villeta, M., Gromov hyperbolic cubic graphs. To appear in Central European J. Math. DOI 10.2478/s11533-012-00364

[25] Portilla, A., Rodríguez, J. M., Sigarreta, J. M. and Vilaire, J.-M., Gromov hyperbolic tessellation graphs. To appear in Utilitas Math.

[26] Portilla, A., Rodríguez, J. M. and Tourís, E., Gromov hyperbolicity through decomposition of metric spaces II, J. Geom. Anal. 14 (2004), 123-149.

[27] Portilla, A., Rodríguez, J. M. and Tourís, E., Stability of Gromov hyperbolicity, J. Advan. Math. Studies 2 (2009), 77-96.

[28] Portilla, A. and Tourís, E., A characterization of Gromov hyperbolicity of surfaces with variable negative curvature, Publ. Mat. 53 (2009), 83-110.

[29] Rodríguez, J. M. and Sigarreta, J. M., Bounds on Gromov hyperbolicity constant in graphs. Proc. Indian Acad. Sci. Math. Sci. 122 (2012), 5365.

[30] Rodríguez, J. M., Sigarreta, J. M., Vilaire, J.-M. and Villeta, M., On the hyperbolicity constant in graphs, Discrete Math. 311 (2011), 211-219.

[31] Rodríguez, J. M. and Tourís, E., Gromov hyperbolicity through decomposition of metric spaces, Acta Math. Hung. 103 (2004), 53-84.

[32] Rodríguez, J. M. and Tourís, E., Gromov hyperbolicity of Riemann surfaces, Acta Math. Sinica 23 (2007), 209-228.

[33] Tourís, E., Graphs and Gromov hyperbolicity of non-constant negatively curved surfaces, J. Math. Anal. Appl. 380 (2011), 865-881.

[34] Wu, Y., Zhang, C., Chordality and hyperbolicity of a graph, Electr. J. Comb. 18 (2011), \#P43. 